\title{
Spectroscopy of Young Planetary Mass Candidates with Disks
}

\author{
Ray Jayawardhana \\ Department of Astronomy $\&$ Astrophysics, University of Toronto, Toronto, ON M5S 3H8, \\ Canada \\ rayjay@astro.utoronto.ca \\ and \\ Valentin D. Ivanov \\ European Southern Observatory, Ave. Alonso de Cordova 310\%, Vitacura, Santiago 19001, \\ Chile \\ vivanov@eso.org
}

\begin{abstract}
It is now well established that many young brown dwarfs exhibit characteristics similar to classical T Tauri stars, including infrared excess from disks and emission lines related to accretion. Whether the same holds true for even lower mass objects, namely those near and below the Deuterium-burning limit, is an important question. Here we present optical spectra of six isolated planetary mass candidates in Chamaeleon II, Lupus I and Ophiuchus star-forming regions, recently identified by Allers and collaborators to harbor substantial mid-infrared excesses. Our spectra, from ESO's Very Large Telescope and New Technology Telescope, show that four of the targets have spectral types in the $\sim$ M9-L1 range, and three of those also exhibit $\mathrm{H} \alpha$. Their luminosities are consistent with masses of 5-15 $\mathrm{M}_{\text {Jupiter }}$ according to models of Chabrier, Baraffe and co-workers, thus placing these four objects among the lowest mass brown dwarfs known to be surrounded by circum-sub-stellar disks. Our findings bolster the idea that freefloating planetary mass objects could have infancies remarkably similar to those of Sun-like stars and suggest the intriguing possibility of planet formation around primaries whose masses are comparable to those of extra-solar giant planets. Another target appears to be a brown dwarf ( M 8 ) with prominent $\mathrm{H} \alpha$ emission, possibly arising from accretion. The sixth candidate is likely a background source, underlining the need for spectroscopic confirmation.
\end{abstract}

Subject headings: accretion, accretion disks — planetary systems — circumstellar matter - stars: formation, low-mass, brown dwarfs 


\section{Introduction}

Thanks to extensive studies conducted in the past five years, it is now clear that many (if not most) young brown dwarfs undergo a classical T Tauri phase similar to that of solar mass stars. Evidence for dusty disks around young sub-stellar objects come from infrared and millimeter excess (e.g., Natta et al. 2002; Jayawardhana et al. 2003; Scholz et al. 2006). Broad and asymmetric emission lines, such as $\mathrm{H} \alpha$, indicate on-going, and often variable, accretion (e.g., Jayawardhana, Mohanty \& Basri 2003; Natta et al. 2004; Mohanty et al. 2005; Scholz et al. 2005). Forbidden line emission, thought to arise in outflows or winds, is also seen in some cases (Fernández \& Comerón 2001; Barrado y Navascués \& Jayawardhana 2004; Whelan et al. 2005). These striking similarities between young low-mass stars and objects near and below the sub-stellar boundary are sometimes taken as evidence for a common formation scenario.

Meanwhile, a few dozen free-floating objects with inferred masses near and below the Deuterium-burning limit of $~ 12$ Jupiter masses (Chabrier et al. 2000a) have been identified in the $\sigma$ Orionis region (Zapatero Osorio et al. 2000) and the Orion Nebula Cluster (Lucas and Roche 2000; Lucas, Roche and Tamura 2005). Low-resolution spectra have confirmed cluster membership and ultra low masses for some of them according to evolutionary models (Barrado y Navascués et al. 2001; Martin et al. 2001; Lucas et al. 2001). These 'isolated planetary mass objects' ('IPMOs', 'planemos') or 'sub-brown dwarfs', as they are sometimes called, represent the bottom end of the stellar initial mass function (IMF). Thus, any successful theory of star formation must account for their origin as well. However, little is known about the properties of 'planemos' because they are very faint at the distances of $\sigma$ Ori and ONC ( 350-450 pc), and only a few more have been identified in closer star forming regions. In particular, it is extremely interesting to investigate whether they also harbor accretion disks, just like many of the higher mass young brown dwarfs. At least in a few cases, there is already evidence of mid-infrared excess (e.g., Testi et al. 2002; Luhman et al. 2005a, 2005b) and strong $\mathrm{H} \alpha$ emission from objects near and below the Deuterium-burning limit (Barrado y Navascués et al. 2001, 2002).

Now, by combining ground-based optical and near-infrared photometry with Spitzer "Cores to Disks" Legacy Survey data, Allers et al. (2006) have identified six new candidate planemos in three nearby regions, at distances $\sim 150$ pc. What's more, based on their infrared excess, these objects appear to be surrounded by dusty disks. However, spectroscopy is essential to determine their true nature, in particular whether they indeed have late spectral types, and thus relatively cool temperatures and very low masses. That is the goal of the present Letter. 


\section{Observations and Data Reduction}

The spectra of targets 1, 5 and 17 were obtained with FORS2 (FOcal Reducer/low dispersion Spectrograph; Appenzeller et al. 1998) at the European Southern Observatory's (ESO) Very Large Telescope on Paranal, and for targets 11, 12 and 18 with EMMI (ESO Multi-Mode Instrument; Dekker, Delabre \& D'Odorico 1986) at the ESO New Technology Telescope on La Silla. The summary observing log is given in Table 1.

The FORS2 observations used grism GRIS_150I+27 with order sorting filter OG590+32, SR collimator, and $1.3^{\prime \prime}$ wide slit, yielding resolution of $\mathrm{R} \sim 260$ over $600-1100 \mathrm{~nm}$ wavelength range. For each target we obtained two spectra of $1347 \mathrm{sec}$ each.

The EMMI observations were carried out in RILD mode, with Grism \#7 and order sorting filter OG530\#645, binning $2 \times 2$ and $1.5^{\prime \prime}$ wide slit, yielding resolution of $\mathrm{R} \sim 280$ over $530-1000 \mathrm{~nm}$ wavelength range. We obtained one $1268 \mathrm{sec}$ exposure of target 11, one $600 \mathrm{sec}$ exposure of target 18, and two $900 \mathrm{sec}$ exposures of target 12 .

The data reduction included the usual steps for long slit optical spectrocopy: first bias subtraction and flat fielding. Next, we extracted 1-dimensional spectra and removed the sky emission lines, interpolating between the sky spectra on both sides of the target spectrum with the IRAF task apall (Tody et al. 1993). After wavelength calibration with arc frames, we corrected for the instrument efficiency with observations of the spectrophotometric standards EG 274 and LTT 6248 (Hamuy et al. 1992, 1994), for FORS2 and for EMMI observations, respectively.

\section{Results}

Fig. 1 shows our spectra of the six candidates, smoothed by a 3-pixel boxcar and dereddened using extinction values derived by Allers et al. (2006) from multi-color photometry and comparison to colors of known young M-type objects. (Their reported uncertainty in $\mathrm{A}_{V}$ is \pm 2 magnitudes.) Five of the objects have spectra consistent with being late-type stars or brown dwarfs, while the sixth (\#12) is most likely a background source, possibly a galaxy or a quasar. In fact, the $I$-band acquisition image shows some nebulosity surrounding it. Based

on its very low luminosity, candidate \#12 was inferred to have a mass of only $7 \mathrm{M}_{\text {Jupiter }}$ if it were a member of the Ophiuchus star-forming region (Allers et al. 2006), but our spectrum shows that it is unlikely to be a sub-stellar object; this result once again underlines that spectroscopy is essential to determine the nature of planetary mass candidates.

For the remaining five candiates, in Fig. 1 we also plot spectra of cool field stars 
from Kirkpatrick et al. (1999) for comparison. This allows us to find the closest match spectral type for each of the candidates by examining a variety of features (e.g., TiO and VO absorption troughs) as well as the overall shape of the spectrum. In practice, we overlaid (scaled) comparison spectra for a number of spectral sub-types along with each target's spectrum and chose the "best-match"; We repeated this procedure with spectra of several standards of each sub-type for each target. However, we note the well known difficulties of deriving precise and reliable spectral types for the coolest young objects, especially since the classification is based on objects with very different surface gravities (e.g., Testi et al. 2002). With these caveats in mind, we report our best-fit spectral types for these five candidates in Table 2. For \#1, \#11 and \#18, we expect our derived classification to be accurate to within about one sub-type, while for \#5 and \#17 the uncertainty may be closer to two sub-types.

In Fig. 2, we show a zoom of the spectral region around $\mathrm{H} \alpha$ for the five late type objects. Three of them (\#01, \#11, \#18) show clear emission. A fourth object (\#05) does have $\mathrm{H} \alpha$ as well, though the detection is less reliable due to low signal-to-noise. The sixth object, \#17, does not show emission in our (noisy) spectrum. Given the poor detection of the continuum in our spectra, it is difficult to measure the $\mathrm{H} \alpha$ equivalent widths (EWs) reliably, so we report minimum EWs in Table 2.

\section{Discussion}

We find that five of the six candidates have spectral types in the late $\mathrm{M}$ to early $\mathrm{L}$ range, as would be expected for low mass brown dwarfs and planetary mass objects, while a sixth (\#12) is probably a background source. Given that the spectral types we derive and the effective temperatures found by Allers et al. from photometry are consistent within the errors, these five objects are likely to be at the distances of the Chamaeleon II, Lupus I and Ophiuchus clouds. Their Spitzer-detected mid-infrared excesses, modeled as emission from dusty disks by Allers et al., provide strong evidence of youth. Here it is interesting to note that the only object in their sample for which Allers and co-workers were not able to fit the spectral energy distribution (SED) well with a flat or flared disk model is \#12, which we now find to be a likely background source. The large minimum $\mathrm{H} \alpha \mathrm{EW}$ s we find in at least three of the five late-type objects bolsters the case for their youth. $\mathrm{H} \alpha$ could originate from disk accretion or chromospheric activity in young low-mass objects (e.g., Jayawardhana, Mohanty \& Basri 2002), and it is often difficult to distinguish definitively between these two possibilities with low-resolution spectra. However, according to the criterion developed by Barrado y Navascués \& Martin (2003), three of these objects, if not four, could well be accreting. The lack of clear $\mathrm{H} \alpha$ emission in \#17 does not necessarily rule out youth; young 
L-type objects may not show $\mathrm{H} \alpha$ unless they are accreting (Barrado y Navascués et al. 2001, 2002).

There are many uncertainties involved in deriving the masses of young very low mass objects, given the difficulties of determining their ages and distances reliably as well as the uncertainties in the spectral type to $\mathrm{T}_{\text {eff }}$ conversion and in the evolutionary models themselves (e.g., Baraffe et al. 2002; Mohanty, Jayawardhana \& Basri 2004). Allers et al. (2006) estimate masses for these candidates by matching their source luminosities to the widely used isochrones of Baraffe et al. (2001; 2003), and assuming ages of 1 Myr for Ophiuchus and Lupus I and 3 Myr for Chamaeleon II. They also point out that three of the objects we have now confirmed as late type (\#01, \#05, \#17) have luminosities equivalent (within the errors) to the lowest luminosity young brown dwarf with mid-infrared excess reported previously (Luhman et al. 2005b).

While we do not think an exhaustive analysis is warranted, given the uncertainties involved, we can at least check whether the spectral types we have derived are roughly consistent with the temperatures derived by Allers et al. from source SEDs. Thus, in Table 2 we report effective temperatures for our targets, based on the spectral type to $\mathrm{T}_{\text {eff }}$ conversion scale of Martin et al. (1999), along with those from Allers et al. The comparison shows that the two sets of derived $\mathrm{T}_{\text {eff }}$ indeed agree within $\pm 100 \mathrm{~K}$ for four out of five objects. For the fifth, \#18, we find a somewhat earlier spectral type, and a correspondingly higher $\mathrm{T}_{\text {eff }}$, than implied by the Allers et al. analysis.

Fig. 3 shows a Hertzsprung-Russell diagram of the five late-type objects. The absolute $K$ magnitude was derived from the apparent $K$ magnitude, the distance modulus and the visual absorption $\left(A_{v}\right)$ given in Allers et al., and adopting the reddening law from Rieke \& Lebofsky (1985). The effective temperatures $\left(T_{\text {eff }}\right)$ are those reported in Table 2 . The isochrones for 1 Myr and 5 Myr DUSTY00 models (Chabrier et al. 2000b; Baraffe et al. 2001) are also plotted. This figure shows that four of the candidates have masses near or below the deuterium-burning limit, while \#18 is a somewhat higher mass brown dwarf. Given the slope of the isochrones around the locus occupied by these objects and the $\approx \pm 100$ $\mathrm{K}$ uncertainty in $T_{\text {eff }}$, this figure suggests an uncertainty in the mass estimates on the order of a few Jupiters for four objects and ${ }_{-5}^{+7}$ for the fifth, \#18. The ages of all five objects are consistent, within the uncertainties, to those assumed by Allers et al.

The targets \#1, \#5, \#11 and \#17 are among the lowest mass objects with dusty disks known to date, and at least three of the four also show possible signs of accretion from those disks. Whatever their exact masses are, these objects represent the bottom end of the stellar IMF. It is worth noting that Mohanty et al. (2006) have recently found evidence for an edge-on disk surrounding the planetary mass companion to the nearby young brown dwarf 
2MASSW J1207334-393254, which itself is known to harbor a disk.

Our findings, combined with previous work, suggest that some planetary mass objects have characteristics usually seen in T Tauri stars and higher mass brown dwarfs, implying strikingly similar infancies for our Sun and objects that are some hundred times less massive. Thus, a successful theory for star formation should be able to account for these similarities in young objects with a wide range of masses.

The shape of the IMF at these lowest masses is not yet well defined observationally. Deep, wide-field optical and infrared surveys with 8-meter class telescopes are needed to investigate this regime with larger samples (e.g., Lucas, Roche \& Tamura 2005). With current observing facilities, spectroscopic confirmation of very low mass candidates is challenging, but not impossible as we have demostrated here (also see Lucas et al. 2001; Barrado y Navascués et al. 2001). As Allers et al. have shown, Spitzer could play a pivotal role in determining the disk frequency in the planetary mass regime.

This paper is based on observations collected at the European Southern Observatory, Chile, under program number 276.C-5050. We thank the ESO staff for carrying out the observations in service mode, Aleks Scholz for useful discussions, and an anonymous referee for suggestions towards improving the manuscript. This research was supported by an NSERC grant and University of Toronto startup funds to RJ.

\section{REFERENCES}

Allers, K.N., Kessler-Silacci, J.E., Cieza, L.A., \& Jaffe, D.T. 2006, ApJ, in press

Appenzeller, I., et al. 1998, The Messenger 94, 1

Barrado y Navascués, D., et al. 2001, A\&A, 377, L9

Barrado y Navascués, D., et al. 2002, A\&A, 395, 813

Barrado y Navascués, D. \& Jayawardhana, R. 2004, ApJ, 615, 840

Barrado y Navascués, D. \& Martìn, E. L. 2003, AJ, 126, 2997

Baraffe, I., et al. 2001, A\&A, 382, 563

Baraffe, I., et al. 2002, A\&A, 382, 563

Baraffe, I., et al. 2003, A\&A, 402, 701 
Chabrier, G., et al. 2000a, ApJ, 542, L119

Chabrier, G., et al. 2000b, ApJ, 542, 464

Dekker, H., Delabre, B. \& D'Odorico, S. 1986, SPIE 627, 39

Fernández, M. \& Comerón, F. 2001, A\&A, 380, 264

Hamuy, M., et al. 1992, PASP, 104, 533

Hamuy, M., et al. 1994, PASP, 106, 566

Jayawardhana, R., Mohanty, S. \& Basri, G. 2002, ApJ, 578, L141

Jayawardhana, R., Mohanty, S. \& Basri, G. 2003, ApJ, 592, 282

Jayawardhana, R., et al. 2003, AJ, 126, 1515

Kirkpatrick, J.D., et al. 1999, ApJ, 519, 802

Lucas, P.W. \& Roche, P.F. 2000, MNRAS, 314, 858

Lucas, P.W., et al. 2001, MNRAS, 326, 695

Lucas, P.W., Roche, P.F. \& Tamura, M. 2005, MNRAS, 361, 211

Luhman, K.L., et al. 2005a, ApJ, 620, L51

Luhman, K.L., et al. 2005b, ApJ, 635, L93

Martin, E.L., et al. 1999, AJ, 118, 2466

Martin, E.L., et al. 2001, ApJ, 558, L117

Mohanty, S., Jayawardhana, R. \& Basri, G. 2004, ApJ, 609, 885

Mohanty, S., Jayawardhana, R. \& Basri, G. 2005, ApJ, 626, 498

Mohanty, S., Jayawardhana, R., Huélamo, N. \& Mamajek, E.E. 2006, ApJ, submitted

Natta, A., et al. 2002, A\&A, 393, 597

Natta, A., et al. 2004, A\&A, 424, 603

Rieke, G.H. \& Lebofsky, M.J. 1985, ApJ, 288, 618

Scholz, A., Jayawardhana, R. \& Brandeker, A. 2005, ApJ, 629, L41 
Scholz, A., Jayawardhana, R. \& Wood, K. 2006, ApJ, 638, 1056

Testi, L., et al. 2002, ApJ, 571, L155

Tody, D., et al. 1993, in Astronomical Data Analysis Software and Systems II, A.S.P. Conference Ser., Vol 52, eds. R.J. Hanisch, R.J.V. Brissenden, \& J. Barnes, 173

Whelan, E.T., et al. 2005, Nature, 435, 652

Zapatero Osorio, M.R., et al. 2000, Science, 290, 103 

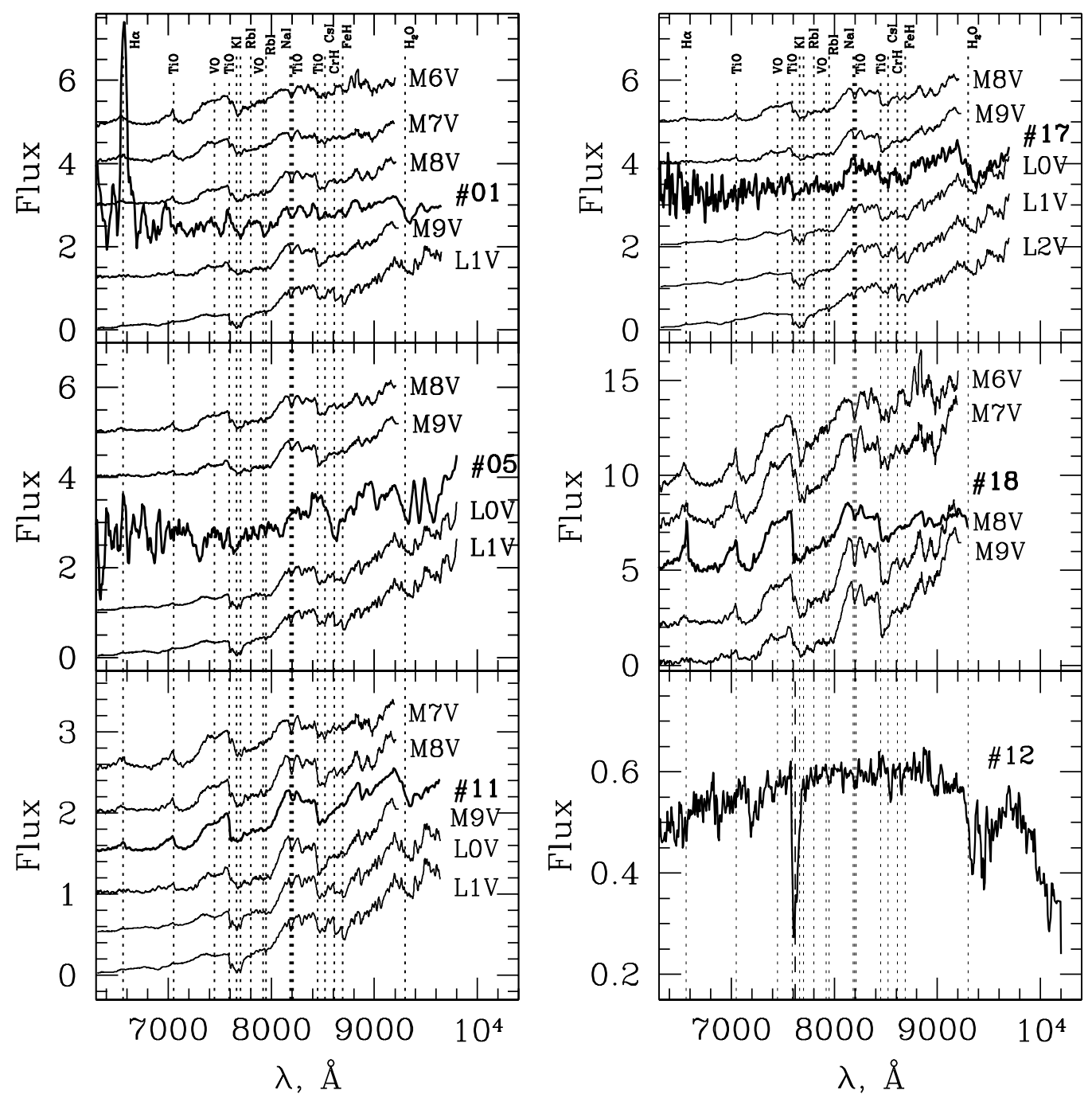

Fig. 1.- Optical spectra of the six 'planemo' candidates, along with those of comparison objects. Dotted lines mark the location of $\mathrm{H} \alpha$ and a number of other features while the dashed line in the lower-right panel corresponds to a telluric absorption feature. 


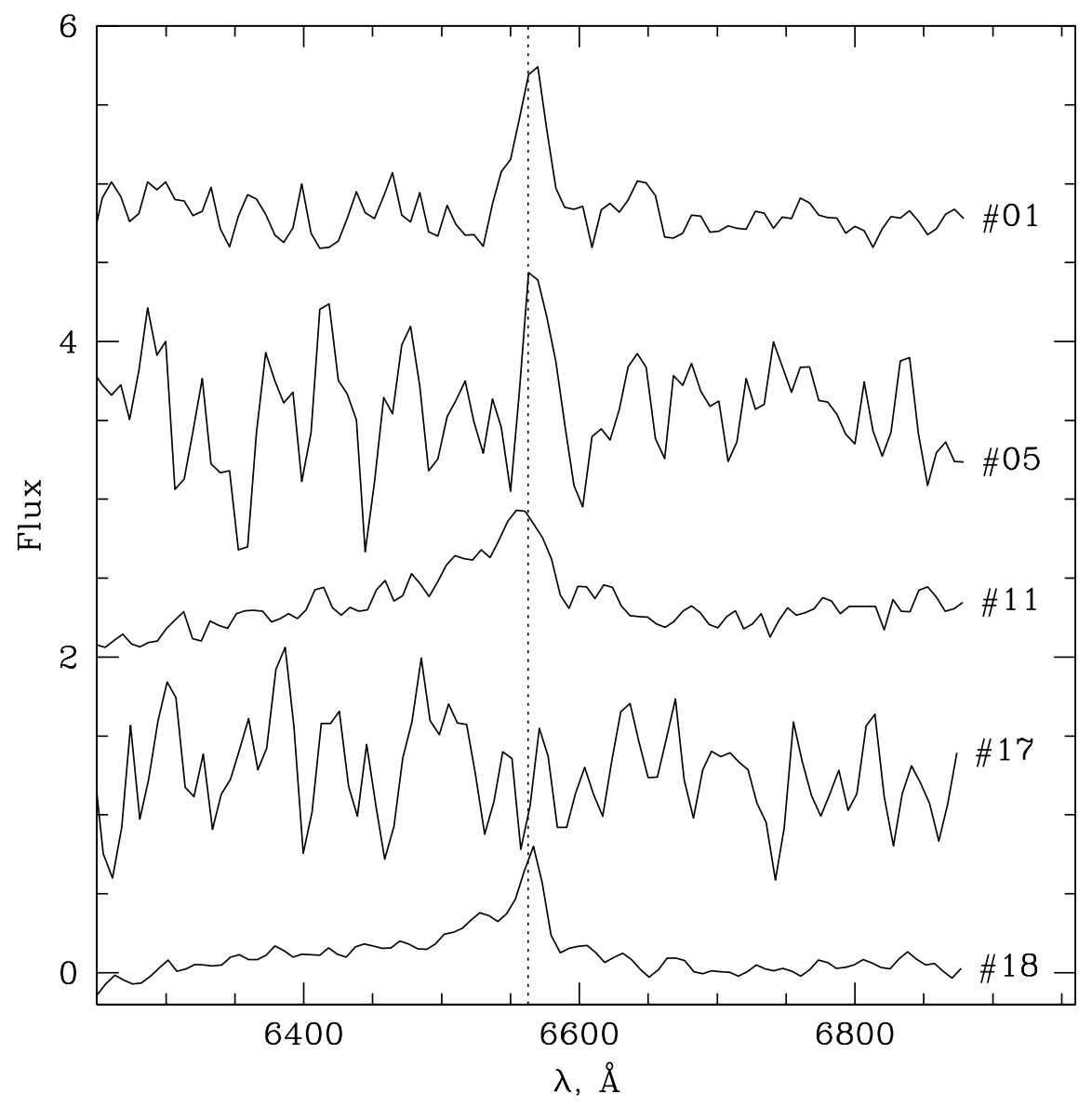

Fig. 2.- Zooming in on the region that includes the $\mathrm{H} \alpha$ line for the five late-type objects. 


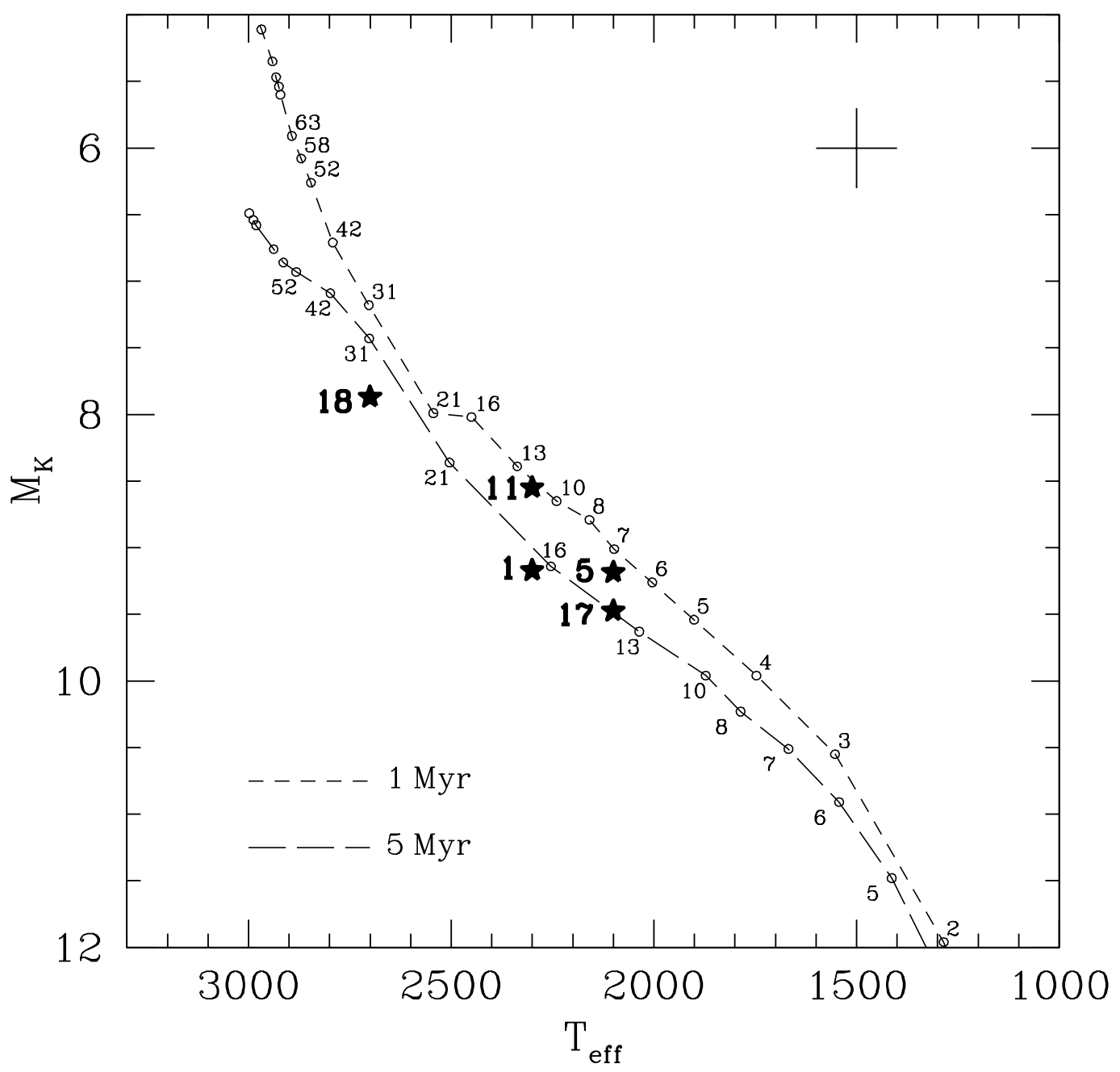

Fig. 3.- Hertzsprung-Russell diagram of the five late-type objects in our sample, labelled according to Allers et al. (2006) notation. Isochrones for 1 Myr (short-dashed line) and 5 Myr (long-dashed line) are plotted, with masses marked in units of Jupiter mass, for DUSTY00 models (Chabrier et al. 2000b; Baraffe et al. 2001). The typical errorbars are shown in the top right corner. The $M_{K}$ uncertainty combines the photometric errors given in Allers et al. with estimates of the errors in the extinctions (0.2 mag in $K$ ) and the distances (15\%), added in quadrature. The typical error in $T_{\text {eff }}$ is estimated to be $\pm 100 \mathrm{~K}$. 
Table 1. Summary Observing Log

\begin{tabular}{cccc}
\hline \hline Target Name & Target ID $^{\mathrm{a}}$ & Date & Start of Observations (UT) \\
\hline Cha 125758-770119 & 01 & $2006-03-15$ & $06: 40: 48.797$ \\
Cha 130540-773958 & 05 & $2006-03-15$ & $07: 41: 12.119$ \\
Oph 162225-240515 & 11 & $2006-03-13$ & $06: 23: 08.854$ \\
Oph 162230-232224 & 12 & $2006-03-13$ & $07: 03: 17.530$ \\
Lup 153927-344844 & 17 & $2006-03-15$ & $08: 41: 38.721$ \\
Lup 154140-334518 & 18 & $2006-03-13$ & $06: 00: 25.611$ \\
\hline
\end{tabular}

a Target ID number is from Allers et al. (2006)

Table 2. Derived Parameters

\begin{tabular}{ccccc}
\hline \hline $\begin{array}{c}\text { Target } \\
\text { ID }\end{array}$ & $\begin{array}{c}\text { Min. } \\
\text { EW }(\mathrm{H} \alpha)\end{array}$ & $\begin{array}{c}\text { Spectral } \\
\text { Type }\end{array}$ & $\begin{array}{c}\mathrm{T}_{\text {eff }} \pm 100 \mathrm{~K} \\
\text { from SpT }\end{array}$ & $\begin{array}{c}\mathrm{T}_{\text {eff }} \mathrm{K} \text { from } \\
\text { Allers et al. (2006) }\end{array}$ \\
\hline 01 & $-90 \AA$ & M9 & 2300 & 2207 \\
05 & $-30 \AA^{\mathrm{a}}$ & L0 & 2100 & 2100 \\
11 & $-50 \AA$ & M9 & 2300 & 2207 \\
17 & - & L0 & 2100 & 2004 \\
18 & $-55 \AA$ & M8 & 2700 & 2400 \\
\hline
\end{tabular}

${ }^{a}$ Due to poor signal-to-noise in this spectrum, the uncertainty in the $\mathrm{EW}(\mathrm{H} \alpha)$ could be as large as $\pm 15 \AA$. 